\title{
Prospective analysis of self-perceived quality of life before and after posterior fossa decompression in 112 patients with Chiari malformation with or without syringomyelia
}

\author{
Diane Mueller, N.D., R.N., C-F.N.P., AND John J. Oro', M.D. \\ Division of Neurosurgery, University Health Care, University of Missouri-Columbia, Missouri
}

\begin{abstract}
Object. The purpose of this prospective study was to determine if there was a difference in the self-perceived quality of life (QOL) before and after surgery among patients with Chiari I malformations with or without syringomyelia. Most patients with Chiari I malformations report improvement in their QOL after decompression surgery; however, specific outcome data have not been empirically studied in this patient population.

Methods. One hundred seventy-two consecutive patients who underwent posterior fossa decompression based on neuroimaging evidence of a Chiari I malformation with or without syringomyelia were prospectively offered participation in the study. The Sickness Impact Profile (SIP) was chosen as the instrument for data collection.

Completed questionnaires were returned by 112 patients. A statistically significant improvement $(\mathrm{p}<0.0001)$ in SIP scores (self-reported QOL) was noted in $84 \%$ of participants after decompression surgery. Patient age, amount of tonsillar herniation, and evidence of syringomyelia before surgery did not correlate with or adversely affect outcomes. Among 16 participants who reported worsening in their QOL, anecdotal information revealed extraneous factors unrelated to the Chiari I malformation that they perceived as negatively influencing their outcome. Among the extraneous variables noted by the participants were general health status, unrelated injury, other illnesses, and significant stress.

Conclusions. The majority of participants who underwent posterior fossa surgery for a Chiari I malformation reported significant improvement in their QOL after surgery.
\end{abstract}

\section{KEY WORDS - Chiari malformation • syringomyelia $\bullet$ syrinx $\bullet$ quality of life • tonsillar herniation}

Chiari malformations are hindbrain anomalies that were first described by Dr. Hans Chiari in 1891 as abnormal findings on postmortem examinations. ${ }^{2,3}$ Three types of Chiari malformations are recognized today. The Chiari I malformation consists of cerebellar tonsillar herniation of 3 to $5 \mathrm{~mm}$ or more below the level of the foramen magnum. The diagnostic test of choice for Chiari I malformation is an MR image of the brain performed without contrast agents. Chiari I malformation is generally diagnosed in the adult population, and is based on MR imaging findings of tonsillar herniation as described earlier. For reasons that are not fully understood, many persons with neuroimaging evidence of Chiari I malformation do not present with symptoms until the fourth or fifth decade of life. Participants in this study presented with symptoms consistent with Chiari I malformation, and all had evidence of this syndrome with or without syringomyelia on MR imaging.

The Chiari II malformation consists of caudally displaced cerebellar tonsils and medulla, with the possible additional features of myelodysplasia and hydrocephalus.

\footnotetext{
Abbreviations used in this paper: $\mathrm{CI}=$ confidence interval; $\mathrm{MR}=$ magnetic resonance; $\mathrm{QOL}=$ quality of life; SIP = Sickness Impact Profile.
}

This type of Chiari malformation is diagnosed at birth or in infancy. The rarest and most severe form of Chiari malformation is the Chiari III type. This is diagnosed at birth and consists of cerebellar structures that are herniated caudally into the upper spinal canal, accompanied by a cervical meningocele and often hydrocephalus. The Chiari III malformation is generally associated with a very poor prognosis.

Symptoms related to Chiari I malformation with or without syringomyelia can be vague and transient. The most common symptom is headache, often in the posterior occipital area, that is frequently triggered or worsened by Valsalva maneuvers. The headache is often described as pressure or 'vice-like' in nature, and can be quite debilitating. Other symptoms include dizziness, pain, weakness, numbness or paresthesia of the face or extremities, difficulty swallowing, fatigue, tinnitus, and memory difficulties. ${ }^{10,11}$

Syringomyelia, although occasionally associated with other causes, is most frequently the result of a Chiari malformation (Type I or II) and is caused by obstruction of cerebrospinal fluid. ${ }^{7}$ The most common symptom of syringomyelia is pain that is described as burning in nature. The pain is generally located in the area of the syrinx (cervical or thoracic); however, it can radiate to the sternum, 
below the scapula, or beneath the ribs. Posterior fossa decompression is the treatment of choice for patients with neuroimaging evidence of Chiari I malformation with or without syringomyelia who experience associated symptoms refractory to conservative treatment. ${ }^{1,12}$

Technical improvements in posterior fossa surgery have had a positive impact on surgery-related morbidity. Most anecdotal information regarding patient outcome implies that overall satisfactory improvement is achieved after decompression surgery. Nevertheless, the influence of the surgical procedure on the individual's perception of his or her functional capacity and QOL has not been empirically studied. The multifaceted concept of the QOL has gained prominence in the field of health care as a fundamental outcome indicator for medical and surgical interventions. The individual's perception of QOL has become increasingly recognized as a key standard by which clinicians and patients alike can make decisions and measure outcomes..$^{5,8,14-16}$ Although the literature is replete with information about the evaluation and treatment of Chiari I malformations, a search of the English literature yielded no prospective empirical analysis of the QOL among patients treated for this syndrome with or without syringomyelia. Thus, the purpose of this prospective study was to determine if there was a difference in the patient's selfperceived QOL before compared with after surgery. Permission to conduct this study was granted by the Institutional Review Board at the University of Missouri Health Care prior to data collection.

\section{CLINICAL MATERIAL AND METHODS Patient Population}

Participation in this prospective study was offered to 172 consecutive patients who electively underwent posterior fossa decompression for Chiari I malformation between 2000 and 2003 at the University of Missouri Health Care (participation was completely voluntary). All participants had evidence of Chiari I malformation on MR images. All patients presented with symptoms consistent with Chiari I malformation, and their disease was refractory to conservative treatments. The most common presenting symptom was posterior occipital headache (97\%). Table 1 lists the 10 most common presenting symptoms in order of frequency among the participants.

Of the 172 patients, 18 declined participation, two died

TABLE 1

Presenting symptoms in 112 patients who underwent posterior fossa decompression for a Chiari malformation

\begin{tabular}{lc}
\hline \hline \multicolumn{1}{c}{ Symptom } & \% of Patients \\
\hline headache & 97 \\
dizziness & 85 \\
neck pain & 78 \\
vision changes & 71 \\
fatigue & 70 \\
difficulty sleeping & 68 \\
tinnitus & 66 \\
extremity numbness & 65 \\
extremity weakness & 65 \\
memory \& concentration problems & 61 \\
\hline
\end{tabular}

of causes unrelated to the Chiari malformation prior to the 1-year follow up, and 40 did not return the 1-year followup survey. Of the 40 patients who did not return the questionnaires, five were lost to follow up. Review of the clinic notes and anecdotal information obtained at follow-up visits ranging from 3 to 6 months postoperatively for the remaining 35 patients revealed that $25(71 \%)$ had improved, seven were unchanged, and three worsened in their symptom reports.

Thus, 112 participants were prospectively studied using data collected from the SIP. The age range of the participants was 17 to 70 years, with a mean age of 40 years (median 39, standard deviation 11.3 years). Eight participants were male and 104 were female. Twenty-two patients $(19.6 \%)$ had neuroimaging evidence of syringomyelia on MR images obtained before surgery. Cerebellar tonsillar herniation ranged from 3 to $30 \mathrm{~mm}$, with a mean herniation of $9.4 \mathrm{~mm}$ (median 9, standard deviation $4.77 \mathrm{~mm}$ ). Seven participants $(6.2 \%)$ had previously undergone posterior fossa decompression at other institutions, and reported persistent symptoms related to the Chiari I malformation that were refractory to conservative treatments. The most common symptom among patients in this group was posterior occipital headache that was unresponsive to conservative treatments. In two participants there was neuroimaging evidence of arachnoid cyst causing caudal displacement of the cerebellar tonsils, resulting in symptoms consistent with Chiari I malformation. Two participants had incidental MR imaging evidence of a small mass lesion with an appearance consistent with meningioma. Nevertheless, the tumors did not appear to affect the position of the cerebellar tonsils. There was neuroimaging evidence of significant spinal scoliosis in three participants with syringomyelia, and another patient without syringomyelia had neuroimaging evidence of KlippelFeil deformity.

\section{Treatment Protocol}

All participants electively underwent standard posterior fossa craniectomy, laminectomy, and pericranial duraplasty. Posterior fossa decompressions were performed with the patient placed prone, with his or her head supported in a three-pin headholder. A midline incision was made from $4 \mathrm{~cm}$ above the inion to $\mathrm{C}-3$ to expose the pericranium above the inion, the suboccipital area, and the C-1 lamina. A $3 \times 3-\mathrm{cm}$ craniectomy and C-1 laminectomy were performed. A $3 \times 4-\mathrm{cm}$ (in some cases $3 \times 5-\mathrm{cm}$ ) triangular pericranial graft was obtained with a needle tip monopolar. The dura mater was opened in the midline at the cervical spinal canal and the incision was extended superiorly in a $Y$-shaped fashion. In the patients with more pronounced herniations, the arachnoid was opened and the tonsils were shrunk with bipolar diathermy. The pericranial graft was anchored at each corner of the open dura mater with 4-0 braided nylon, and each side was closed with a running 5-0 monofilament suture. Before closure, the subarachnoid space was filled with saline in patients in whom the arachnoid had been opened. After dural closure, two Valsalva maneuvers were performed to ensure absence of a spinal fluid leak, and if leakage was present, the incision was closed with additional suture and/or fascia from the wound. Generally, a sheet of Avitine Ultrafoam 
was laid over the dura and graft, and was mixed with a small amount of the patient's own local wound blood to create a blood patch. The wound was closed in layers with absorbable sutures and skin staples. All surgical procedures were performed by the senior author (J.O.). No syrinx shunts were placed.

\section{Postoperative Complications}

Among the participants, there were no postoperative hemorrhages, strokes, cranial nerve deficits, or pseudomeningoceles. Three patients $(2.6 \%)$ experienced postoperative occipital neuralgia. In one participant a posterior fossa arachnoid cyst developed after decompression, and she required an additional operation. The patient recovered well and experienced no further complications. On the 1-year follow-up survey, she rated her QOL and general health as improved. Two of the participants $(1.8 \%)$ had superficial wound infections postoperatively, which were treated with antibiotics without further sequelae.

\section{Survey Methods}

The multidimensional concept of QOL involves information regarding the physical, psychosocial, emotional, and functional capacity of the individual. The SIP has been used since 1976 to measure outcomes of health care interventions. ${ }^{4,6,13}$ The instrument is a self-reported patient questionnaire that includes the domains of physical, psychosocial, recreation, sleep, work, and social interaction. A self-report questionnaire has been documented to be the most accurate form of data collection to measure an individual's QOL. ${ }^{9}$ The questionnaire contains 127 items pertaining to such activities as rest, sleep, depressive ideation, movement, balance, hygiene, home maintenance, social interaction, concentration, communication, recreation, and nutrition. Anecdotal information provided by patients with Chiari I malformation indicated that these were the realms of daily living and QOL that were most adversely affected by their symptoms; therefore, this instrument was chosen for the current study. We had no financial interest in the use of this instrument. Written permission for its use was obtained from the authors of the SIP prior to data collection.

The research design used an interrupted time-series, self-assessment survey to test the perception of QOL before and after surgical decompression for Chiari I malformation. An interrupted time-series design was chosen because the participants were queried preoperatively and again at 1 year postsurgery. The higher the score, the greater the self-perceived impairment, whereas the lower scores indicated minimal impairment. Participation in the study was voluntary and in no way affected the choice of surgical procedure or the care provided.

All participants received the questionnaire along with a cover letter explaining the study 30 days before surgery. The patients returned the preoperative questionnaire to the principal investigator (D.M.) before surgery. An identical, blank questionnaire was then mailed to each patient on the 1-year anniversary of surgery; these were completed and returned by mail. The 1-year follow-up questionnaire also included open questions regarding the patients' perception of their QOL since surgery, their perception of their general health status, and their activity status, which was rated as better, worse, or the same since surgery. In addition, an open-ended question was included that asked patients to describe their perception of their QOL since surgery and to report any factors that they believed had influenced their QOL after surgery.

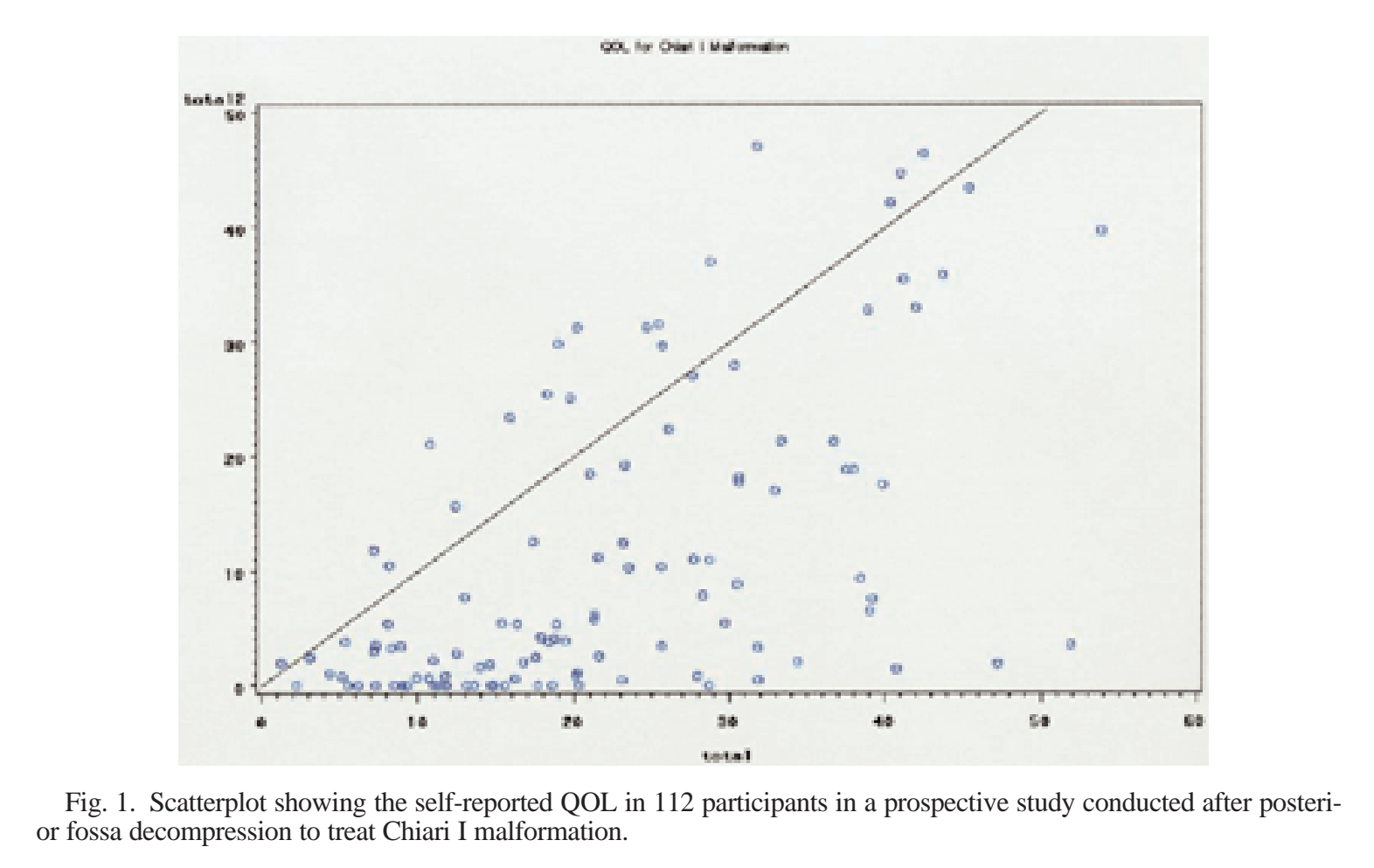




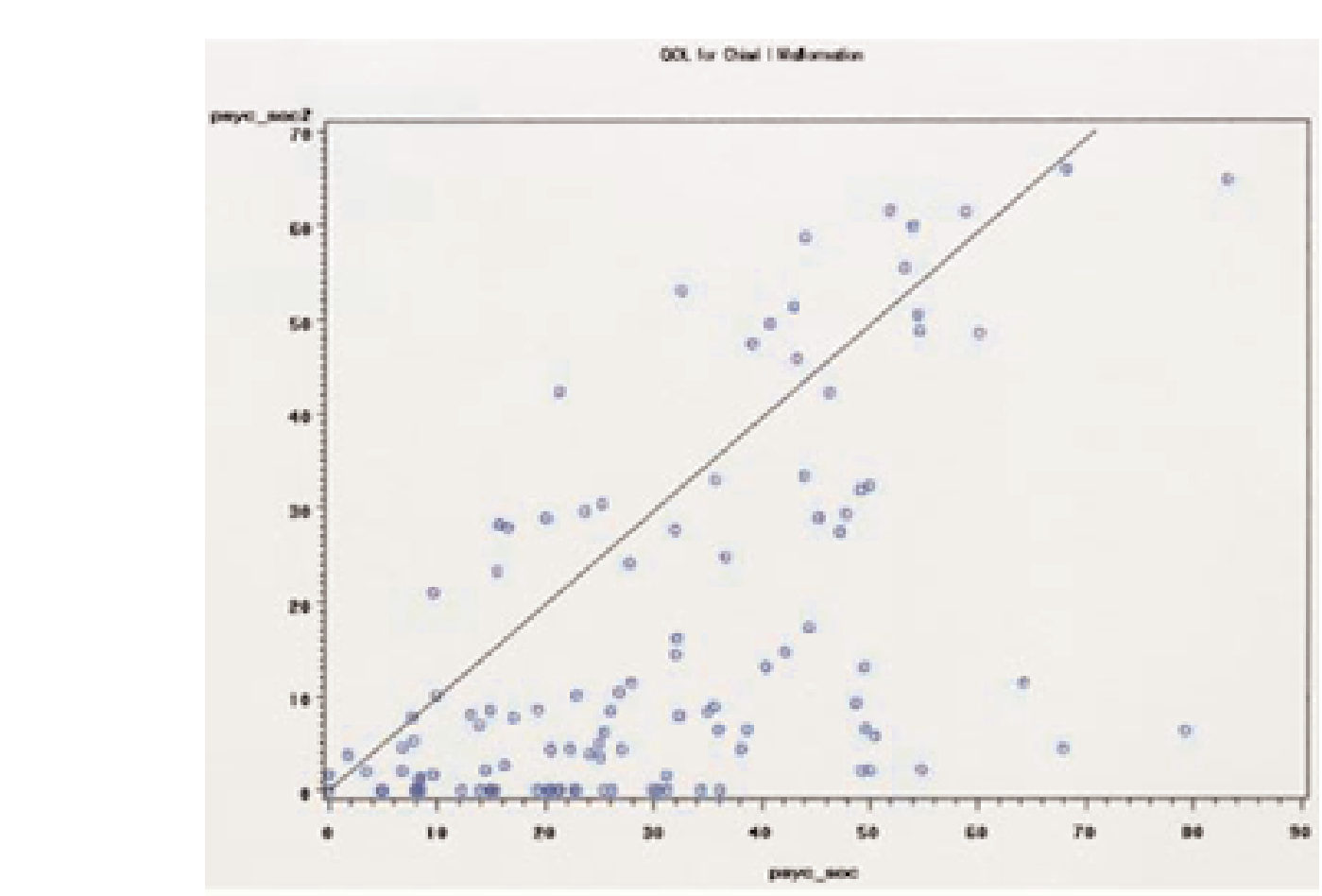

Ca la Oes i Wdontin

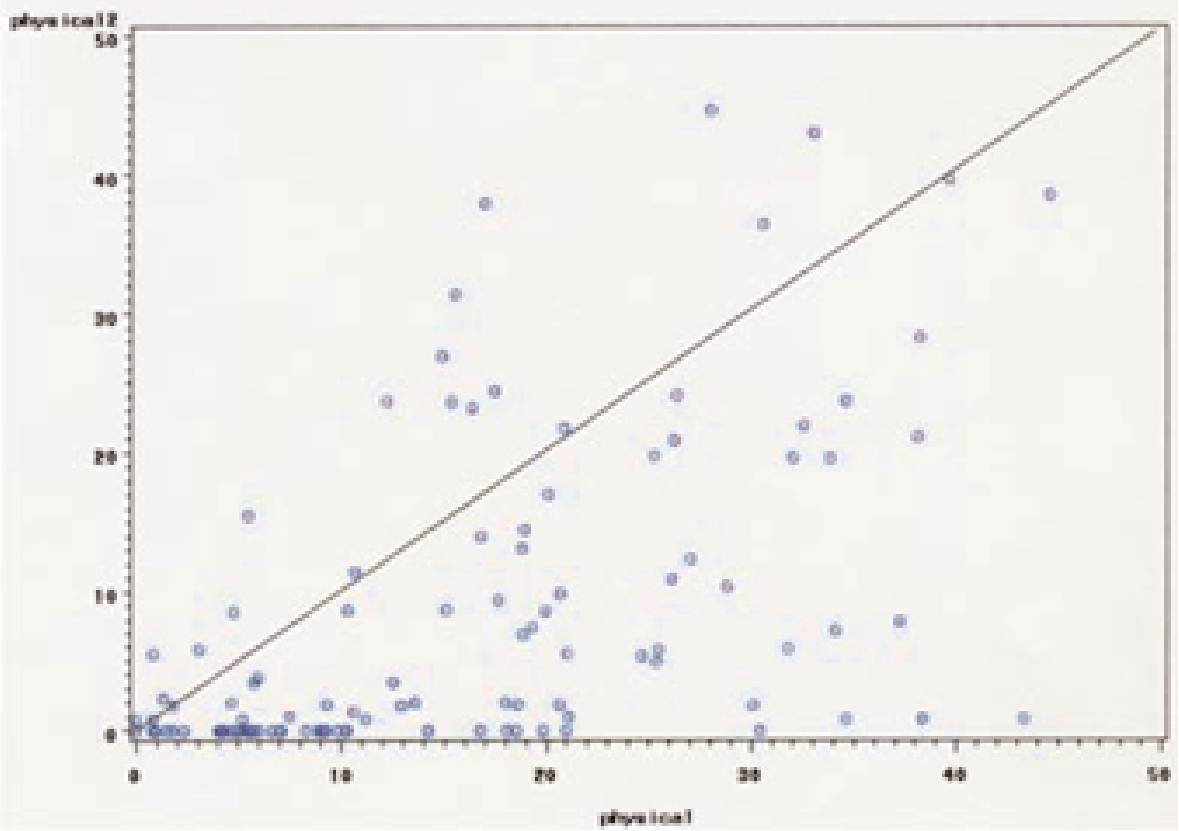

Fig. 2. Scatterplots showing the scores in the psychosocial (upper) and physical (lower) realms of the SIP in 112 patients who participated in the QOL study.

\section{RESULTS}

A total of 112 questionnaires were analyzed. Each patient served as his or her own control for pre- and postoperative comparison. The primary research question asked if there was a difference in self-perceived QOL before surgery compared with afterward. Based on results of the Wilcoxon signed-rank test, a highly statistically signifi- cant improvement $(\mathrm{p}<0.0001)$ was noted in the QOL after surgery compared with before the procedure. This indicated that $84 \%$ of participants $(95 \% \mathrm{CI})$ reported significant improvement in QOL after decompression surgery (Fig. 1). The highest (worst) total preoperative score was 528.8 , and the lowest (best) postoperative score was 0 . Improvement on the psychosocial scale was 79\% (p < 
$0.0001,95 \% \mathrm{CI}$ ), whereas the total physical scale improved by $77 \%(\mathrm{p}<0.0001,95 \% \mathrm{CI})$. There were no statistical differences in scores when comparing the physical and psychosocial realms (Fig. 2). All of the subscales showed statistically significant improvement. There was no statistically significant difference in patients with syringomyelia before surgery compared with those in whom syringomyelia had not been identified. There was no correlation (not significantly different from zero) between the amount of tonsillar herniation and the degree of improvement after surgery.

Among the seven participants who were undergoing their second surgical decompression, six reported a significant improvement in their QOL after reoperation. One patient reported worse headaches after the repeated surgery.

Review of the open-ended questions revealed that 84 participants (75\%) rated their QOL as improved after surgery, $17(15.2 \%)$ reported no change, and $11(9.8 \%)$ reported worsening in their status. Many patients added anecdotal information, such as the statements "I have my life back," "I was able to return to work," "I wish I had done the surgery sooner," and "I did not realize how sick I was before."

General health was reported as improved in 87 participants $(77 \%)$, unchanged in $12(11 \%)$, and worsened in 13 (12\%). Among the 13 participants who reported worsening of their general health and QOL, 10 offered anecdotal information regarding their perception of why they believed their condition had worsened. None of the participants related Chiari I malformation or the surgery as the possible cause of their unchanged or worsened QOL and general health. One participant reported other medical problems, one underwent extensive lumbar fusion, Epstein-Barr virus was diagnosed in one, two suffered workrelated injuries, severe depression was diagnosed in one, another reported persistent occipital neuralgia after surgery, one underwent excision of recurrent brain tumor, one described "unbearable pressure at work," and one was involved in a motor vehicle crash. Three patients did not comment on the cause of their worsened QOL status.

\section{DISCUSSION}

The results of this study indicate that there is a significant improvement in the patient's self-perceived QOL after posterior fossa decompression for Chiari I malformation. The amount of tonsillar herniation had no influence on the outcome after surgery. Patients with syringomyelia did not have statistically different outcomes compared with those in whom syringomyelia was not found preoperatively. Age was also not an influential factor in outcome; patients older than 65 years also reported significant improvement in their QOL. As expected, other extraneous factors also influenced the patient's perception of the QOL. Although these factors were unavoidable, general health issues, incidental trauma, and significant stress adversely affected outcome for patients in this cohort.

The research data indicated a positive outcome for patients who underwent surgical intervention for Chiari I malformation. The design of this study did not include evaluation of the outcome for those patients who elected conservative management of their symptoms. Research should be undertaken to compare 1-year outcomes in patients who elect surgical decompression with those who choose medical management.

One limitation of the study was the loss of five participants to initial follow up and the 35 patients who failed to return their survey. The $62 \%$ satisfactory improvement noted on review of the records and early clinical followup findings in these 35 patients, if added to the $84 \%$ who experienced improvement in QOL in the 112 participants reported, would yield a $79 \%$ improvement in all patients.

\section{CONCLUSIONS}

From this sample it was found that the majority of persons with Chiari I malformation, with or without syringomyelia, benefit from posterior fossa decompression in terms of their self-perceived QOL, as measured 1 year postsurgery. Generalizability, however, is limited because all participants in this cohort were from a group of patients evaluated and treated at the University of Missouri, Columbia. Variability in surgical methods and postoperative care may influence outcomes. A multicenter study design may aid in generalizability of the data.

\section{Acknowledgments}

We gratefully acknowledge Dr. Richard Madsen, Professor Emeritus of Biostatistics, and Ms. Ashley Sherman, statistician, Department of Biostatistics, University of Missouri, Columbia for statistical consultation and analysis. We also gratefully acknowledge all of the patients who volunteered to participate in this project.

\section{References}

1. Alzate JC, Kothbauer KF, Jallo GI, et al: Treatment of Chiari I malformation in patients with and without syringomyelia: a consecutive series of 66 cases. Neurosurg Focus 11(1):E3, 2001

2. Anson JA, Benzel EC, Awad IA (eds): Syringomyelia and the Chiari Malformations. Park Ridge, IL: AANS, 1997

3. Bejjani GK: Definition of the adult Chiari malformation: a brief historical overview. Neurosurg Focus 11(1):E1, 2001

4. Bergner M, Bobbitt RA, Carter WB, et al: The Sickness Impact Profile: development and final revision of a health status measure. Med Care 19:787-805, 1981

5. Gil Z, Abergel A, Spektor S, et al: Quality of life following surgery for anterior skull base tumors. Arch Otolaryngol Head Neck Surg 129:1303-1309, 2003

6. Gilson BS, Gilson JS, Bergner M, et al: The Sickness Impact Profile. Development of an outcome measure of health care. Am J Public Health 65:1304-1310, 1975

7. Guyotat J, Bret P, Jouanneau E, et al: Syringomyelia associated with type I Chiari malformation. A 21-year retrospective study on 75 cases treated by foramen magnum decompression with a special emphasis on the value of tonsils resection. Acta Neurochir 140:745-754, 1998

8. Laxton AW, Perrin RG: The relations between social support, life stress, and quality of life following spinal decompression surgery. Spinal Cord 41:553-558, 2003

9. Leplege A, Hunt S: The problem of quality of life in medicine. JAMA 278:47-50, 1997

10. Milhorat TH, Chou MW, Trinidad EM, et al: Chiari I malformation redefined: clinical and radiographic findings for 364 symptomatic patients. Neurosurgery 44:1005-1017, 1999 


\section{Mueller and J. J. Oro'}

11. Mueller DM, Oro' JJ: Prospective analysis of presenting symptoms among 265 patients with radiographic evidence of Chiari malformation type I with or without syringomyelia. J Am Acad Nurse Pract 16:134-138, 2004

12. Nash J, Cheng JS, Meyer GA, et al: Chiari type I malformation: overview of diagnosis and treatment. WMJ 101:35-40, 2002

13. Pollard WE, Bobbitt RA, Bergner M, et al: The Sickness Impact Profile: reliability of a health status measure. Med Care 14: 146-155, 1976

14. Schrag A, Jahanshahi M, Quinn N: What contributes to qualify of life in patients with Parkinson's disease? J Neurol Neurosurg Psychiatry 69:308-312, 2000

15. Shah S, Har-El G, Rosenfeld RM: Short-term and long-term quality of life after neck dissection. Head Neck 23:954-961, 2001

16. Staal C, Arends A, Ho S: A self-report of quality of life of patients receiving intrathecal baclofen therapy. Rehabil Nurs 28: 159-163, 2003

Manuscript received September 23, 2004.

Accepted in final form January 5, 2005.

Address reprint requests to: Diane Mueller, N.D., R.N., CF.N.P., Division of Neurosurgery, University Health Care, University of Missouri-Columbia, Columbia, Missouri 65212. email: muellerdm@health.missouri.edu. 